\title{
On the geometry of proportional quotients of $l_{1}^{m}$
}

\author{
by \\ Piotr Mankiewicz (Warszawa) and \\ Staniseaw J. Szarek (Paris and Cleveland)
}

\begin{abstract}
We compare various constructions of random proportional quotients of $l_{1}^{m}$ (i.e., with the dimension of the quotient roughly equal to a fixed proportion of $m$ as $m \rightarrow \infty)$ and show that several of those constructions are equivalent. As a consequence of our approach we conclude that the most natural "geometric" models possess a number of asymptotically extremal properties, some of which were hitherto not known for any model.
\end{abstract}

1. Introduction. The geometry of random quotients and subspaces of finite-dimensional Banach spaces (or equivalently, random projections and sections of convex bodies) has been studied by several authors (cf., e.g., [S1], [Mi2], [MiS], [G], [S2], [M2], [Bo], [MT2], [MT4]). Typical results in this direction can be rephrased as the following dichotomy (or rather a 0-1 law):

Given a geometric property of normed spaces, either a vast majority of quotients (or subspaces) of a fixed dimension of a given Banach space enjoy that property, or a similar vast majority strongly violate it.

Examples of such properties include having a "well-bounded" basis constant, volume ratio or the Banach-Mazur distance to a Hilbert space, to name a few.

Results of this type require introduction of a measure on quotients (or subspaces) with respect to which the "vast majority" is to be meant. There are two different ways of imposing such a measure, even if the differences may seem to be unessential. The first one, which we call geometric, is based on introducing a suitable scalar product on the space $X$ in question (usually induced by one of the classical ellipsoids connected with the unit ball of $X$ ),

2000 Mathematics Subject Classification: 46B07, 46B09, 46B20.

Research of the first author was supported in part by KBN grant no. 2 P03A 01319. Research of the second author was supported in part by grants from the National Science Foundation (U.S.A.). Research of both authors was supported in part by an international cooperation grant from Min. Foreign Aff.-France \& KBN-Poland. 
which leads to the Haar measure on the Grassmann manifold of kernels of the quotient maps (or the subspaces). The geometric approach is arguably most natural; one of its desirable features is that the results obtained refer directly to the intrinsic geometry of the underlying space $X$ (cf., e.g., [Mi1], [K], [FKP], [S1], [Mi2], [MiS]). The other approach, which we call probabilistic, stems from the ground-breaking result of Gluskin on the diameters of the Minkowski compacta and is based on introducing on quotients (or subspaces) of the space $X$ a suitable probability, usually given by some random matrix. The main advantage of this method lies in the fact that by an appropriate choice of the probability structure it is possible to exploit tools such as independence, which greatly simplifies arguments. This approach has led a number of authors to some very strong, often asymptotically optimal results for which, till very recently, no counterparts in the geometric setting have been known (cf. [G], [S2], [S4], [M2]). For more information on the subject we refer the reader to [MT3].

The distinction between the two approaches is admittedly not very clearcut. On the one hand, the Grassmann manifold endowed with the normalized Haar measure becomes a probability space. On the other hand, considering as the quotient map, e.g., an $n \times m$ (with $n<m$ ) random matrix whose entries are independent identically distributed (i.i.d.) Gaussian random variables leads to a random $n$-dimensional normed space which has the same distribution as that given by the geometric model (if one identifies isometric spaces). This follows from the fact that the distribution of such a random matrix does not change if we compose it on the right (or, for that matter, on the left) with an orthogonal matrix, and so the distribution of the kernels of the random quotients (which do determine the quotients up to isometry) on the corresponding Grassmannian is invariant under the action of the orthogonal group, hence the same as the one given by the Haar measure. However, the proofs of the strongest results mentioned above would not, in general, carry over to this particular probabilistic model. For example, in some probabilistic constructions, the basis constant of a typical proportional quotient of $l_{1}^{m}$ was of the maximal order (i.e., $\sqrt{m}$; see [S2]). An analogous result (our Corollary 2.3) was not known in the geometric setting. In the opposite direction, it is not difficult to deduce from [S4] that in the geometric setting the DvoretzkyRogers factorization constant of an $n$-dimensional quotient of $l_{1}^{2 n}$ is greater than or equal to $c(n / \log n)^{1 / 10}$, for a "vast majority" of $n$-dimensional quotients, where $c>0$ is a numerical constant. On the other hand, it is quite obvious that in some probabilistic settings, like those in [G] or [S2], a "vast majority" of such quotients admit Dvoretzky-Rogers factorization bounded by, say, 2. Thus the two methods have led to quite different conclusions. 
The aim of the present paper is to show that the differences between the two approaches described at the beginning of the previous paragraph are essentially technical and that most of known asymptotically optimal results for the proportional quotients of $l_{1}^{m}$ in the probabilistic setting remain valid in the geometric context. In particular, the historically first (to our knowledge) family of random $n$-dimensional quotients of $l_{1}^{2 n}$ from [FKP] does a posteriori have such extremal properties. Our arguments use, in particular, a technique developed in [MT4] to study the behavior of Banach-Mazur distances between random projections of arbitrary symmetric convex bodies.

Our main technical result, Theorem 2.1, states that in the geometric setting a vast majority of $n$-dimensional quotients $Y$ of $l_{1}^{\lfloor(1+\delta) n\rfloor}$ have the property that for all sufficiently nontrivial operators (i.e. for $(\kappa n, 1)$-mixing operators, see the paragraph containing (2.1) for a definition) in $L(Y)$ the norms $\|T x\|_{Y}$ are greater than $c \sqrt{n}$ for at least $\delta n$ extreme points of the unit ball of $Y$, with $c=c(\kappa, \delta)>0$ depending on $\kappa$ and $\delta$ only. This immediately implies that the basis constants for such quotients are greater than $c(1 / 2, \delta) \sqrt{n} / 2$ (cf. Corollary 2.3). Another application of this theorem says that for such $Y$ 's and for an arbitrary sufficiently nontrivial compact group of operators $G \subset L(Y)$ one has

$$
\text { Average } \int_{G}\|T x\|_{X} d h_{G}(T) \geq c^{\prime} \sqrt{n},
$$

where the average is taken over all extreme points of the unit ball of $Y$, $h_{G}$ stands for the normalized Haar measure on $G$ and $c^{\prime}$ depends on some parameters describing how nontrivial the group $G$ is. Finally, note that these estimates are slightly better (by a logarithmic factor) than those previously known even for the probabilistic case [M2].

2. Main results. We shall use standard notation of local theory of Banach spaces (cf., e.g., [T]). For $m \in \mathbb{N},\left\{e_{i}\right\}_{i=1}^{m}$ will stand for the standard unit vector basis in the Euclidean space $\mathbb{R}^{m}$ while $\|\cdot\|_{p}$ (for $\left.p \in[1, \infty]\right)$ will denote the $l_{p}$-norm. For a Banach space $X=\left(\mathbb{R}^{m},\|\cdot\|_{X}\right)$ we shall denote by $B_{X}$ its unit ball; in the special case of $X=l_{p}^{m}:=\left(\mathbb{R}^{m},\|\cdot\|_{p}\right)$, we will write simply $B_{p}^{m}$. In what follows we shall identify Banach spaces with their unit balls, e.g., the norm of a linear operator $T$ acting between the spaces whose unit balls are, respectively, $U$ and $V$ may be denoted by $\|T: U \rightarrow V\|$ and the space of such operators endowed with that norm by $L(U, V)$.

For $n, m \in \mathbb{N}$ with $n<m, \mathbf{G}_{m, n}$ is the Grassmann manifold of $n$ dimensional (linear) subspaces of $\mathbb{R}^{m}$ endowed with the (unique) normalized measure $h_{m, n}$ invariant with respect to the action of the orthogonal group $\mathbf{O}_{m}$. For $E \in \mathbf{G}_{m, n}$, set $B_{E}:=P_{E}\left(B_{1}^{m}\right)$, where $P_{E}$ stands for the orthogonal projection onto $E$. Denote by $\|\cdot\|_{B_{E}}$ the norm induced 
on $E$ by $B_{E}$ and let $X_{E}:=\left(E,\|\cdot\|_{B_{E}}\right)$. Obviously, each such $X_{E}$ is an $n$-dimensional quotient of $l_{1}^{m}$ (specifically $X_{E}=l_{1}^{m} / E^{\perp}$ ) and its unit ball is $B_{E}=\operatorname{symconv}\left\{u_{i}(E) \mid i=1, \ldots, m\right\}$, where $u_{i}(E):=P_{E}\left(e_{i}\right)$ for $i=1, \ldots, m$. We shall refer to these quotients - with measure induced by $h_{m, n}$-as to (the family of) geometric quotients of $l_{1}^{m}$. We point out that in addition to the normed space structure (which depends only on the kernel of the quotient map, $E^{\perp}$ ), this representation endows $E$ with a Euclidean structure inherited from $\mathbb{R}^{m}$.

If $K \subset \mathbb{R}^{n}$, let $\operatorname{ext}(K)$ denote the set of extreme points of $K$. Clearly, for every $E \in \mathbf{G}_{m, n}, \operatorname{ext}\left(B_{E}\right)=\operatorname{ext}\left(P_{E}\left(B_{1}^{m}\right)\right) \subset\left\{ \pm u_{i}(E) \mid i=1, \ldots, m\right\}$.

Recall that for a Hilbert space $H, k \leq \operatorname{dim} H / 2$ and $\beta>0$ an operator $T \in L(H)$ is said to be $(k, \beta)$-mixing if there exists a subspace $E \subset H$ with $\operatorname{dim} E \geq k$ such that

$$
\operatorname{dist}(T x, E)=\left\|P_{E^{\perp}} T x\right\|_{2} \geq \beta\|x\|_{2} \quad \text { for every } x \in E,
$$

where $P_{E^{\perp}}$ stands for the orthogonal projection of $H$ onto $E^{\perp}$. The set of all $(k, \beta)$-mixing operators in $L\left(\mathbb{R}^{n}\right)$ will be denoted by $\operatorname{Mix}_{n}(k, \beta)$.

The following theorem, which constitutes our main technical result, describes the properties of proportional geometric quotients of $l_{1}^{m}$ in terms of norms of mixing operators. In a sense, it improves Theorem 1.4 of [S3] (cf. also Th. 12 in [MT3]).

Theorem 2.1 (Main Technical Result). For every $\delta>0$ and $\kappa \in(0,1 / 2]$ there exists $c=c(\kappa, \delta)>0$ such that for every $\beta>0, n \in \mathbb{N}$ with $n>$ $\max \left\{N_{0}, 8 \kappa^{-1}, 8 \delta^{-1}\right\}$ and $m=\lfloor(1+\delta) n\rfloor$ we have

$$
\begin{array}{r}
h_{m, n}\left\{E \in \mathbf{G}_{m, n} \mid \operatorname{ext}\left(B_{E}\right)=\left\{ \pm u_{i}(E) \mid i=1, \ldots, m\right\}\right. \text { and } \\
\operatorname{card}\left\{i \in\{1, \ldots, m\} \mid\left\|T\left(u_{i}(E)\right)\right\|_{B_{E}} \geq c(\kappa, \delta) \beta \sqrt{n}\right\} \geq \delta n / 2 \\
\text { for every } \left.T \in \operatorname{Mix}_{n}(\kappa n, \beta)\right\} \\
\geq 1-e^{-n},
\end{array}
$$

where $u_{i}(E)=P_{E} e_{i}, i=1, \ldots, m$, and $N_{0}=N_{0}(\delta, 4)$ is taken from Fact 3.3.

REMARK 2.2. In what follows we shall denote by $\mathcal{A}_{m, n, \kappa}$ the subset of $\mathbf{G}_{m, n}$ implicitly defined in (2.2). Thus $h_{m, n}\left(\mathcal{A}_{m, n, \kappa}\right) \geq 1-e^{-n}$. In fact, by a suitable modification of the proof of Theorem 2.1 and an appriopriate choice of $c(\kappa, \delta)$, one can get an estimate on measure of the form $1-e^{-A n}$ for an arbitrary preassigned $A>0$. The same observation holds true for the estimate in Theorem 4.1, the "probabilistic" counterpart of Theorem 2.1.

The proof of Theorem 2.1 will be postponed to the last two sections. It will be based on proving a corresponding statement for a suitably defined class of Gaussian quotients and showing that both models (i.e., geometric and Gaussian) are equivalent. 
Since, historically, many classical parameters of Banach spaces were related to mixing properties of operators (see [MT3, Section 5] for details and references), Theorem 2.1 has many immediate consequences. A sample such consequence is the result on basis constants mentioned in the introduction. Recall that, for a (Schauder) basis $\left(x_{i}\right)$ of a normed space $X$, its basis constant is defined by $\mathrm{bc}\left(x_{i}\right):=\sup _{1<k<\operatorname{dim} X}\left\|P_{k}\right\|$, where $P_{k}$ is the $k$ th partial sum projection with respect to the basis $\left(x_{i}\right)$, and the basis constant of $X$ is $\operatorname{bc}(X):=\inf \operatorname{bc}\left(x_{i}\right)$, with the infimum taken over all bases of $X$. We have

Corollary 2.3. In the notation of Theorem 2.1, for any $E \in \mathcal{A}_{m, n, 1 / 4}$ and for any basis of $X:=l_{1}^{m} / E^{\perp}$,

$$
\underset{x \in \operatorname{ext}\left(B_{X}\right)}{\operatorname{Ave}} \frac{1}{n} \sum_{k=1}^{n}\left\|P_{k} x\right\|_{X} \geq \frac{1}{2} c(1 / 4, \delta) \sqrt{n} .
$$

In particular, $\mathrm{bc}(X) \geq \frac{1}{2} c(1 / 4, \delta) \sqrt{n}$.

This follows immediately from Theorem 2.1 and the well-known fact that a rank $k$ projection on $\mathbb{R}^{n}$ is $(\min \{k, n-k\}, 1 / 2)$-mixing (cf., e.g., [MT3]).

For a vector space $H$, we shall denote by $\mathcal{G}(H)$ the set of all compact groups of (linear) operators acting on $H$ and, for $G \in \mathcal{G}(H)$, the normalized Haar measure on $G$ will be denoted by $h_{G}$. We shall say that such a $G$ is $(k, \beta, p)$-mixing for some $k \leq \operatorname{dim} H / 2, \beta>0$ and $p \in(0,1)$ if

$$
h_{G}\{T \in G \mid T \text { is }(k, \beta) \text {-mixing }\} \geq p .
$$

The following consequence of Theorem 2.1 improves the estimate in [M2, Th. 2.4], and solves Problem 1 therein.

THEOREM 2.4. For every $\delta>0$ and $\kappa \in(0,1 / 2]$ there exists $\widetilde{c}=\widetilde{c}(\kappa, \delta)$ $>0$ such that for every $\beta, p>0, n \in \mathbb{N}$ with $n>\max \left\{N_{0}, 8 \kappa^{-1}, 8 \delta^{-1}\right\}$, $m=\lfloor(1+\delta) n\rfloor$, and every subspace $E \in \mathcal{A}_{m, n, \kappa}$ we have

$$
\underset{x \in \operatorname{ext}\left(B_{E}\right)}{\operatorname{Ave}} \int_{G}\|T x\|_{B_{E}} d h_{G}(T) \geq \widetilde{c} \beta p \sqrt{n}
$$

for every $(\kappa n, \beta, p)$-mixing group $G \in \mathcal{G}(E)$.

Proof. The argument is straightforward, but we will include it for completeness. Let $\kappa \in(0,1 / 2]$. Fix an arbitrary $(\kappa n, \beta, p)$-mixing compact group $G \subset L\left(\mathbb{R}^{n}\right)$ and let

$$
B_{\kappa, \beta}=\left\{T \in G \mid T \in \operatorname{Mix}_{n}(\kappa n, \beta)\right\} .
$$

Then, by Theorem 2.1, for every $E \in \mathcal{A}_{m, n, \kappa}$ we have 


$$
\begin{aligned}
\underset{x \in \operatorname{ext}\left(B_{E}\right)}{\operatorname{Ave}} \int_{G}\|T x\|_{B_{E}} d h_{G}(T) & =(2 m)^{-1} \int_{G} \sum_{x \in \operatorname{ext}\left(B_{E}\right)}\|T x\|_{B_{E}} d h_{G}(T) \\
& \geq(2 m)^{-1} \int_{B_{\kappa, \beta}} \sum_{x \in \operatorname{ext}\left(B_{E}\right)}\|T x\|_{B_{E}} d h_{G}(T) \\
& \geq(2 m)^{-1} \int_{B_{\kappa, \beta}} \delta n c(\kappa, \delta) \beta \sqrt{n} d h_{G}(T) \\
& \geq \frac{\delta n}{2\lfloor(1+\delta) n\rfloor} c(\kappa, \delta) \beta p \sqrt{n},
\end{aligned}
$$

and the theorem follows from $(2.2)$ with $\widetilde{c}(\kappa, \delta)=\delta c(\kappa, \delta) /(2(1+\delta))$.

Now we shall present several applications of Theorem 2.4 to the situations where the mixing properties of the groups involved follow from their algebraic properties, and are independent of the choice of the inner product on the space itself. The first one will concern estimates for compact groups of operators which act irreducibly on a "large" subspace while the second will provide estimates for random unconditional constants for a "typical" proportional quotient of $l_{1}^{m}$. (We leave to the interested reader the even more direct applications to questions like those considered in [S3].)

Recall that a compact group of operators $G \subset L(H)(2<\operatorname{dim} H<\infty)$ is said to act irreducibly on $H$ if any $S \in L(H)$ satisfying $S T=T S$ for all $T \in G$ is of the form $S=\lambda \operatorname{Id}_{H}$ for some $\lambda \in \mathbb{R}$. We shall say that a compact group $G \subset L(H)$ acts $r$-irreducibly for some $r \leq \operatorname{dim} H$ if there exists a $G$-invariant linear subspace $F \subset H$ with $\operatorname{dim} F \geq r$ such that the group $G_{F}:=\left\{T_{\mid F} \mid T \in G\right\} \subset L(F)$ acts irreducibly on $F$.

The first application of Theorem 2.4 generalizes [M2, Theorem 3.2].

Theorem 2.5. For every $\varrho \in(0,1), \delta>0, n>\max \left\{N_{0}, 160 \varrho^{-1}, 8 \delta^{-1}\right\}$, $m=\lfloor(1+\delta) n\rfloor$, every linear subspace $E \in \mathcal{A}_{n, m, \varrho / 20}$ and every compact group of operators $G \subset L(E)$ acting @n-irreducibly on $E$ we have

$$
\begin{aligned}
\sup _{\|x\|_{B_{E}}=1} \int_{G}\|T x\|_{B_{E}} d h_{G}(T) & \geq \underset{x \in \operatorname{ext}\left(B_{E}\right)}{\operatorname{Ave}} \int_{G}\|T x\|_{B_{E}} d h_{G}(T) \\
& \geq \frac{1}{20} \widetilde{c}(\varrho / 20, \delta) \sqrt{n},
\end{aligned}
$$

where $\widetilde{c}(\cdot, \cdot)$ is taken from Theorem 2.4 .

Proof. Clearly, it suffices to prove the second inequality in (2.3). By Theorem 2.4, that inequality will follow if we show that every compact group $G \subset L(E)$ acting $\varrho n$-irreducibly on $E$ is $(\varrho n / 20,1 / 4,1 / 5)$-mixing. To this end, fix such a group $G$ and let $F$ be a $G$-invariant subspace of $E$ with $\operatorname{dim} F \geq \varrho$ such that $G_{F}$, the restriction of $G$ to $F$, acts irreducibly on $F$. 
Then

$$
\begin{aligned}
h_{G}\{T \in G \mid T \text { is } & (\varrho n / 20,1 / 4) \text {-mixing }\} \\
& \geq h_{G}\left\{T \in G \mid T_{\mid F} \text { is }(\varrho n / 20,1 / 4) \text {-mixing }\right\} \\
& =h_{G_{F}}\left\{S \in G_{F} \mid S \text { is }(\varrho n / 20,1 / 4) \text {-mixing }\right\} \geq 1 / 5,
\end{aligned}
$$

the equality following from the invariance of the Haar measures involved and the last inequality from irreducibility of $G_{F}$ combined with [M2, Th. $3.1]$; it remains to compare the first and the last lines.

Recall that the symmetric constant $\operatorname{sym}(X)$ for a finite-dimensional space $X=\left(X,\|\cdot\|_{X}\right)$ is defined by

$$
\operatorname{sym}(X):=\inf _{G} \sup _{T \in G}\|T\|=\inf _{G} \sup _{\|x\|_{X}=1} \sup _{T \in G}\|T x\|_{X}
$$

where the infimum is taken over all compact groups of operators $G \subset L(X)$ acting irreducibly on $X$. Since the geometry of a quotient of a Banach space depends on the kernel of the quotient map only, one can easily deduce from Theorem 2.5 a generalization of the main result of [M1].

Corollary 2.6. Let $n, \delta, m$ and $\widetilde{c}$ be as in Theorem 2.4. Then, for every $E \in \mathcal{A}_{m, n, 1 / 20}$,

$$
\operatorname{sym}\left(l_{1}^{m} / E^{\perp}\right) \geq \frac{1}{20} \widetilde{c}(n / 20, \delta) \sqrt{n} .
$$

Moreover

$$
\underset{x \in \operatorname{ext}\left(l_{1}^{m} / E^{\perp}\right)}{\operatorname{Ave}} \int_{G}\|T x\|_{l_{1}^{m} / E^{\perp}} d h_{G}(T) \geq \frac{1}{20} \widetilde{c}(n / 20, \delta) \sqrt{n}
$$

for every compact group $G$ acting irreducibly on $l_{1}^{m} / E^{\perp}$.

The "moreover" part of Corollary 2.6 provides affirmative answers to Problems 2 and 3 in [M2].

Before passing to the next application of Theorem 2.4, recall that for an $n$-dimensional Banach space $X$ the random unconditional constant $\operatorname{ruc}(X)$ is defined as follows (cf. [BKPS], see also [M2]). For every basis $\left\{x_{i}\right\}_{i=1}^{n}$ of $X$ with dual functionals $\left\{x_{i}^{*}\right\}_{i=1}^{n}$ set

$$
G_{\left\{x_{i}\right\}_{i=1}^{n}}:=\left\{T \in L(X) \mid T=\sum_{i=1}^{n} \varepsilon_{i} x_{i}^{*}(\cdot) x_{i} \text { for some }\left(\varepsilon_{i}\right) \in\{-1,1\}^{n}\right\} .
$$

Clearly, $G_{\left\{x_{i}\right\}_{i=1}^{n}}$ is group-isomorphic to $\mathbb{Z}_{2}^{n}$ and its Haar measure $h_{G_{\left\{x_{i}\right\}_{i=1}^{n}}}$ is the normalized counting measure. We set

$$
\operatorname{ruc}(X):=\inf \sup _{\|x\|_{X}=1} \int_{G_{\left\{x_{i}\right\}_{i=1}^{n}}}\|T x\|_{X} d h_{G_{\left\{x_{i}\right\}_{i=1}^{n}}}(T),
$$

where the infimum is taken over all bases $\left\{x_{i}\right\}_{i=1}^{n}$ of $X$. 
The following fact is an easy consequence of Theorem 2.4 above and of Theorem 3.3 in [M2], and provides an affirmative answer to Problem 4 in the latter. It also strengthens a result from $[\mathrm{B}]$ concerning random unconditional constants of quotients of $l_{1}^{m}$.

Theorem 2.7. There exists $p_{0}>0$ such that for any $\delta>0$, any $n \in \mathbb{N}$ with $n>\max \left\{N_{0}, 32,8 \delta^{-1}\right\}, m=\lfloor(1+\delta) n\rfloor$ and any $E \in \mathcal{A}_{m, n, 1 / 20}$ we have

$$
\underset{x \in \operatorname{ext}\left(l_{1}^{m} / E^{\perp}\right)}{\operatorname{Ave}} \int_{G_{\left\{x_{i}\right\}_{i=1}^{n}}^{n}}\|T x\|_{l_{1}^{m} / E^{\perp}} d h_{G_{\left\{x_{i}\right\}_{i=1}^{n}}}(T) \geq p_{0} \widetilde{c}(1 / 4, \delta) \sqrt{n}
$$

for every basis $\left\{x_{i}\right\}_{i=1}^{n}$ in $l_{1}^{m} / E^{\perp}$, where $\widetilde{c}(1 / 4, \delta)$ is taken from Theorem 2.4 . In particular, for any such $E$,

$$
\operatorname{ruc}\left(l_{1}^{m} / E^{\perp}\right) \geq p_{0} \widetilde{c}(1 / 4, \delta) \sqrt{n} .
$$

3. Gaussian quotients. Let $(\boldsymbol{\Omega}, \mathbf{P})$ be a (sufficiently nontrivial) probability space. For a fixed $m \in \mathbb{N}$, we shall denote by $G_{m}=G_{m}(\omega)$ a (random) $m \times m$ matrix with independent Gaussian $N(0,1 / m)$-distributed entries. We shall often think of $G_{m}$ as a random linear operator on $\mathbb{R}^{m}$. Consequently, the columns of $G_{m}$

$$
h_{i}=h_{i}(\omega):=G_{m}\left(e_{i}\right), \quad 1 \leq i \leq m,
$$

are independent Gaussian $N\left(0, m^{-1} \operatorname{Id}_{\mathbb{R}^{m}}\right)$-distributed vectors in $\mathbb{R}^{m}$.

If $m, n \in \mathbb{N}$ with $n \leq m$, we shall identify $\mathbb{R}^{n}$ with the subspace of $\mathbb{R}^{m}$ spanned by the first $n$ vectors of the basis $\left\{e_{i}\right\}_{i=1}^{m}$. Moreover, for a subset $S \subset\{1, \ldots, m\}$, we shall denote by $\mathbb{R}^{S}$ the linear subspace spanned by $\left\{e_{i} \mid i \in S\right\}$, and $P_{n}$ (resp., $\left.P_{S}\right)$ will stand for the orthogonal projection from $\mathbb{R}^{m}$ onto $\mathbb{R}^{n}$ (resp., $\mathbb{R}^{S}$ ).

Since the (normalized) Haar measure on $\mathbf{G}_{m, n}$ is induced by the (normalized) Haar measure on the orthogonal group $\mathbf{O}_{m}$, the distribution of the family of $n$-dimensional geometric quotients of $l_{1}^{m}$, i.e., $P_{E}\left(B_{1}^{m}\right), E \in \mathbf{G}_{m, n}$, is the same as the distribution of the family $P_{n} U\left(B_{1}^{m}\right), U \in \mathbf{O}_{m}$ (recall that quotients depend, up to isometry, on the kernels of the quotient maps only; the identification $U^{*} P_{n} U=P_{E}$ then does the job). Since the matrices $G_{m}$ behave in many respects similarly to (random) orthogonal matrices (cf., e.g., [S5, Lemma 2.4] for a statement and some proofs and references), this suggests calling $P_{n} G_{m}\left(B_{1}^{m}\right)$ the family of $n$-dimensional Gaussian quotients of $l_{1}^{m}$. The random quotient maps $\Gamma_{n, m}=\Gamma_{n, m}(\omega):=P_{n} G_{m}(\omega), \omega \in \Omega$, are exactly the $n \times m$ random matrices with i.i.d. Gaussian entries mentioned in the introduction, and it can be readily verified (in many ways) that the distribution of their kernels is uniform in $\mathbf{G}_{m, m-n}$. Accordingly, the distribution of the Gaussian quotients $l_{1}^{m} / \operatorname{ker} \Gamma_{n, m}$ (as Banach spaces) with respect to $\mathbf{P}$ is the same as that of the geometric ones with respect to $h_{m, n}$. 


\section{Define}

$$
g_{i}(\omega)=\Gamma_{n, m}\left(e_{i}\right)=P_{n} h_{i}(\omega)
$$

for $\omega \in \boldsymbol{\Omega}$ and $i=1, \ldots, m$ and set

$$
B_{n, m, \omega}=\Gamma_{n, m}(\omega)\left(B_{1}^{m}\right)=\operatorname{symconv}\left\{g_{i}(\omega) \mid i=1, \ldots, m\right\} .
$$

We shall need three auxiliary results on Gaussian operators. For the sake of future references we shall state them in a slightly more general version than needed in the present argument. In all three facts we shall deal with tail estimates of the form $e^{-A n}$ while in what follows we shall use them for $A=4$ only. The first fact is a direct consequence of Fact 1.5 in [MT4].

FACT 3.1. For every $\delta, A>0$ there exists $c=c(\delta, A)$ such that for every $n \in \mathbb{N}$ and $m=\lfloor(1+\delta) n\rfloor$ we have

$$
\begin{array}{r}
\mathbf{P}\left\{\left.\omega \in \boldsymbol{\Omega}|| \operatorname{det}\left(\Gamma_{n, m \mid \mathbb{R}^{S}}\right)\right|^{1 / n} \geq c(\delta, A)\right. \\
\quad \text { for every } S \subset\{1, \ldots, m\} \text { with card } S=n\} \geq 1-e^{-A n} .
\end{array}
$$

Proof. Since the columns of $\Gamma_{n, m}$ are identically distributed, hence exchangeable, the distribution of $\left|\operatorname{det}\left(\Gamma_{n, m \mid \mathbb{R}^{S}}\right)\right|$ is the same for all $\left(\begin{array}{c}m \\ n\end{array}\right)$ subsets $S \subset\{1, \ldots, m\}$ with card $S=n$. Next, specifying $S=\{1, \ldots, n\}$, we note that the (random) operator $\Gamma_{n, m \mid \mathbb{R}^{n}}: \mathbb{R}^{n} \rightarrow \mathbb{R}^{n}$ has the same distribution as $\sqrt{n / m} G_{n}$. It is thus enough to analyze $\operatorname{det}\left(G_{n}\right)$ for which, by Fact 1.5 in [MT4], we have

$$
\mathbf{P}\left\{\left.\omega \in \boldsymbol{\Omega}|| \operatorname{det}\left(G_{n}\right)\right|^{1 / n} \geq c_{0} \kappa^{2}\right\} \geq 1-\kappa^{n}
$$

for every $\kappa \in(0,1)$, where $c_{0}>0$ is a suitable numerical constant. Choosing $\kappa=e^{-(1+\delta+A)}$ and noting that then $\left(\begin{array}{c}m \\ n\end{array}\right) \kappa^{n}<e^{-A n}$, we obtain the required estimate with $c(\delta, A)=c_{0}(1+\delta)^{-1 / 2} e^{-2(1+\delta+A)}$.

For fixed $n, m \in \mathbb{N}$ with $n<m$ and $\omega \in \boldsymbol{\Omega}$ we write $\Gamma_{n, m}(\omega)=V_{\omega} P_{E_{\omega}}$ where $P_{E_{\omega}}$ is the orthogonal projection onto $E_{\omega}:=\operatorname{ker} \Gamma_{n, m}(\omega)^{\perp}$ and $V_{\omega}$ : $E_{\omega} \rightarrow \mathbb{R}^{n}$. Note that, with probability $1, \operatorname{dim} E_{\omega}=n$. In fact much more is true: $V_{\omega}$ is typically a "good isomorphism" from $\left(E_{\omega},\|\cdot\|_{2}\right)$ onto $\mathbb{R}^{n}$.

FACT 3.2. For every $\delta, A>0$ there are $c^{\prime}(\delta, A)>0$ and $C=C(A)$ such that

$$
\begin{aligned}
\mathbf{P}\{\omega \in \boldsymbol{\Omega} \mid & \operatorname{rank} V_{\omega}=n \text { and } \\
& \left.c^{\prime}(\delta, A)\|x\|_{2} \leq\left\|V_{\omega} x\right\|_{2} \leq C(A)\|x\|_{2} \text { for every } x \in E_{\omega}\right\} \geq 1-e^{-A n}
\end{aligned}
$$

for every $n \in \mathbb{N}$ and $m=\lfloor(1+\delta) n\rfloor$.

Proof. This is essentially Lemma 2.8 in [S4] except for two "cosmetic" improvements. First, the estimate on the probability there is of the form $1-$ $e^{-a n}$ for some $a>0$. However, a rudimentary modification of the argument yields a similar estimate with $1-e^{-A n}$, for any preassigned $A>0$. Second, to replace the constant in the upper estimate for $\left\|V_{\omega} x\right\|_{2}$ by a function 
providing the required tail estimate it suffices to use Theorem 2.11 of [DS]. For an elementary argument leading to the estimate of the probability of $\left\|V_{\omega}\right\|_{2} \leq 12$ which is sufficient for our purposes (i.e., for $A=4$ ) we refer the reader to [MT3].

The last auxiliary result asserts that unit balls of random quotients of $l_{1}^{m}$ have "typically" exactly $2 m$ extreme points. It is not central to our discussion, but we include it as it allows simplifying some of the statements.

FACT 3.3. For every $\delta, A>0$ there exists $N_{0}=N_{0}(\delta, A)>0$ such that

$$
\mathbf{P}\left\{\omega \in \boldsymbol{\Omega} \mid \operatorname{ext}\left(B_{n, m, \omega}\right)=\left\{ \pm g_{i}(\omega) \mid i=1, \ldots, m\right\}\right\} \geq 1-e^{-A n}
$$

for every $n \geq N_{0}(\delta, A)$ and $m=\lfloor(1+\delta) n\rfloor$.

Proof. This is a simple consequence of several known results. Fix $\delta, A>0$ and $n \in \mathbb{N}$ with $m=\lfloor(1+\delta) n\rfloor>n$. It follows from the definition of the unit balls $B_{n, m, \omega}$ that $\operatorname{ext}\left(B_{n, m, \omega}\right) \subset\left\{ \pm g_{i}(\omega) \mid i=1, \ldots, m\right\}$, and that equality holds unless one of the $g_{i}$ 's belongs to the symmetric convex hull of $n$ others. By exchangeability of $g_{i}$ 's, the probability of that event does not exceed

$$
\left(\begin{array}{c}
m \\
n
\end{array}\right)(m-n) \mathbf{P}\left\{g_{m}(\omega) \in \operatorname{conv}\left\{ \pm g_{i} \mid 1 \leq i \leq n\right\}\right\} .
$$

Next, for any fixed values of $g_{1}, \ldots, g_{n}$, the conditional probability

$$
\begin{aligned}
\mathbf{P}\left\{g_{m}(\omega) \in \operatorname{conv}\left\{g_{i} \mid 1 \leq i \leq n\right\} \mid g_{i}(\omega)\right. & \left.=a_{i} \text { for } 1 \leq i \leq n\right\} \\
\leq & \frac{e^{n / 2} \operatorname{vol}\left(\operatorname{conv}\left\{ \pm a_{i} \mid i \leq n\right\}\right)}{\operatorname{vol} B_{2}^{n}}
\end{aligned}
$$

by [MT3, Fact 1(iv)]. Further, by Fact 3.2, we have $\left\|g_{i}(\omega)\right\|_{2} \leq C(2 A)$ for $1 \leq i \leq n$ on a subset $\boldsymbol{\Omega}^{\prime} \subset \boldsymbol{\Omega}$ with $\mathbf{P}\left(\boldsymbol{\Omega}^{\prime}\right) \geq 1-e^{-2 A n}$. For $\omega \in \boldsymbol{\Omega}^{\prime}$ it follows just from the Hadamard inequality that

$$
\operatorname{vol}\left(\operatorname{conv}\left\{ \pm a_{i} \mid i \leq n\right\}\right) \leq \frac{(2 C(2 A))^{n}}{n !} .
$$

Combining the last three formulae with the estimate on $\mathbf{P}\left(\boldsymbol{\Omega}^{\prime}\right)$, the formula for vol $B_{2}^{n}$ and the Stirling formula yields Fact 3.3.

4. The Gaussian version of the Main Technical Result. The following is a restatement of the "geometric" Theorem 2.1 for the "probabilistic" family of Gaussian quotients of $l_{1}^{m}$. Let $\Omega_{0} \subset \boldsymbol{\Omega}$ denote the intersection of the sets considered in Facts 3.1-3.3 for $A=4$. Then we have

TheOREM 4.1. For every $\delta>0$ and $\kappa \in(0,1 / 2]$ there exists $c^{\prime}=$ $c^{\prime}(\kappa, \delta)>0$ such that for every $\beta>0, n \in \mathbb{N}$ with $n>\max \left\{N_{0}, 8 \kappa^{-1}, 8 \delta^{-1}\right\}$ and $m=\lfloor(1+\delta) n\rfloor$ we have 
$\mathbf{P}\left\{\omega \in \boldsymbol{\Omega}_{0} \mid \operatorname{card}\left\{i \in\{1, \ldots, m\} \mid \| T\left(g_{i}(\omega) \|_{B_{n, m, \omega}} \geq c^{\prime} \beta \sqrt{n}\right\} \geq \delta n / 2\right.\right.$ for every $\left.T \in \operatorname{Mix}_{n}(\kappa n, \beta)\right\} \geq 1-e^{-n}$,

where $g_{i}(\omega)=\Gamma_{n, m} e_{i}$ for $i \in\{1, \ldots, m\}$ and $N_{0}=N_{0}(\delta, 4)$ is taken from Fact 3.3.

In the present section we will explain why Theorems 2.1 and 4.1 are (essentially) equivalent. The rather formal argument is based on Fact 3.2. Then we will reduce the proof of Theorem 4.1 to Proposition 4.2, which will be shown in the next section.

Proof of Theorem 4.1 $\Rightarrow$ Theorem 2.1. Fix $n, \delta$ and $\kappa$ as in Theorem 4.1. Due to the uniform distribution of the kernels of $\Gamma_{n, m}$ and by the uniqueness of the normalized Haar measure we have, for every Borel subset $\mathcal{A} \subset \mathbf{G}_{m, m-n}$,

$$
\begin{aligned}
\mathbf{P}\left\{\omega \in \boldsymbol{\Omega} \mid \operatorname{ker} \Gamma_{n, m}(\omega) \in \mathcal{A}\right\} & =h_{m, m-n}(\mathcal{A}) \\
& =h_{m, n}\left\{E \in \mathbf{G}_{m, n} \mid E^{\perp} \in \mathcal{A}\right\} .
\end{aligned}
$$

Let $\boldsymbol{\Omega}_{n, m, \kappa}$ be the set of $\omega$ 's appearing in Theorem 4.1 and let

$$
\widetilde{\mathcal{B}}_{n, m, \kappa}:=\left\{F \in \mathbf{G}_{m, m-n} \mid F=\operatorname{ker} \Gamma_{n, m}(\omega) \text { for some } \omega \in \boldsymbol{\Omega}_{n, m, \kappa}\right\}
$$

and $\mathcal{B}_{n, m, \kappa}:=\left\{E \in \mathbf{G}_{m, n} \mid E^{\perp} \in \widetilde{\mathcal{B}}_{n, m, \kappa}\right\}$. Thus, by (4.1), we have

$$
h_{m, n}\left(\mathcal{B}_{n, m, \kappa}\right)=h_{m, m-n}\left(\widetilde{\mathcal{B}}_{n, m, \kappa}\right) \geq \mathbf{P}\left(\boldsymbol{\Omega}_{n, m, \kappa}\right) \geq 1-e^{-n} .
$$

Hence it is enough to show that every $E \in \mathcal{B}_{n, m, \kappa}$ satisfies the second condition in Theorem 2.1 with some fixed $c(\kappa, \delta)>0$. (The first condition concerning the extreme points is automatically satisfied since the equality $\operatorname{ext}\left(B_{n, m, \omega}\right)=\left\{ \pm g_{i}(\omega) \mid i=1, \ldots, m\right\}$ is implicit in the definition of $\boldsymbol{\Omega}_{0}$, hence in that of $\mathcal{B}_{n, m, \kappa}$.) To this end, fix an arbitrary $E \in \mathcal{B}_{n, m, \kappa}$ and pick $\omega_{0} \in \boldsymbol{\Omega}_{n, m, \kappa}$ with

$$
E_{\omega_{0}}=\operatorname{ker} \Gamma_{n, m}\left(\omega_{0}\right)^{\perp}=E
$$

and a $(\kappa n, \beta)$-mixing operator $T \in L(E)$. Consider the operator $T^{\prime}=$ $V_{\omega_{0}} T V_{\omega_{0}}^{-1}: \mathbb{R}^{n} \rightarrow \mathbb{R}^{n}$, where $V_{\omega_{0}}$ is the operator from Fact 3.2 . It follows directly from the definition of mixing operators and Fact 3.2 that $T^{\prime} \in \operatorname{Mix}_{n}\left(\kappa n, c^{\prime}(\delta) \beta / 5\right)$. Hence, by Theorem 4.1,

$$
\operatorname{card}\left\{i \in\{1, \ldots, m\} \mid\left\|T^{\prime} g_{i}(\omega)\right\|_{B_{n, m, \omega}} \geq c(\kappa, \delta) \beta \sqrt{n}\right\} \geq \delta n / 2,
$$

where $c(\kappa, \delta)=c^{\prime}(\delta) c^{\prime}(\kappa, \delta) / 5$. The proof is completed by observing that since

$$
g_{i}\left(\omega_{0}\right)=\Gamma_{n, m}\left(\omega_{0}\right)\left(e_{i}\right)=V_{\omega_{0}} P_{E}\left(e_{i}\right)=V_{\omega_{0}} u_{i}(E)
$$

for every $i=1, \ldots, m$, the map $V_{\omega_{0}}$ is an isometry from $\left(E,\|\cdot\|_{B_{E}}\right)$ onto $\left(\mathbb{R}^{n},\|\cdot\|_{B_{n, m, \omega_{0}}}\right)$ and therefore $\left\|T^{\prime} g_{i}\left(\omega_{0}\right)\right\|_{B_{n, m, \omega_{0}}}=\left\|T u_{i}(E)\right\|_{B_{E}}$. 
The proof that Theorem $2.1 \Rightarrow$ Theorem 4.1 (with a small loss in the coefficient in the exponent) goes along the same lines and is left to the reader. However, if both theorems are considered in the version proposed in Remark 2.2, then they are formally equivalent (i.e., the equivalence follows from "general principles," with Fact 3.2 being the only "technical result" used).

In what follows, to avoid excessive use of indices we shall fix $n, \delta$ and $m$ satisfying the hypotheses of the Theorem, and write $B_{\omega}$ (resp., $\|\cdot\|_{\omega}$ ) instead of $B_{n, m, \omega}$ (resp., $\|\cdot\|_{B_{n, m, \omega}}$ ).

Observe that since $\operatorname{Mix}_{n}(\kappa n, \beta) \subset \operatorname{Mix}_{n}\left(\kappa^{\prime} n, \beta\right)$ for every $0<\kappa^{\prime}<\kappa \leq$ $1 / 2$ and $\beta>0$, without any loss of generality we may and will assume that $\kappa \leq \delta$. Also, due to the homogeneity of the norm it suffices to prove Theorem 4.1 for $\beta=1$.

For $d \in \mathbb{N}$ we set $S_{d}=\{1, \ldots, d\}$ and $\widetilde{S}=\{n+1, \ldots, n+\lfloor\kappa n / 4\rfloor\}$. For $\omega \in \boldsymbol{\Omega}$ we let $R(\omega)$ be the orthogonal projection in $\mathbb{R}^{n}$ with $\operatorname{ker} R(\omega)=$ $\operatorname{lin}\left\{g_{i}(\omega) \mid i \in \widetilde{S}\right\}$. Theorem 4.1 is a consequence of the following

Proposition 4.2. For every $\delta>0$ and $\kappa \in(0,1 / 2]$ there exists $c^{\prime}=$ $c^{\prime}(\kappa, \delta)$ such that

$$
\begin{aligned}
& \mathbf{P}\left\{\omega \in \boldsymbol{\Omega}_{0} \mid \text { there exists } T \in \operatorname{Mix}_{n}(\kappa n, 1)\right. \text { such that } \\
& \left.\qquad R(\omega) T g_{i} \in c^{\prime} \sqrt{n} R(\omega) B_{\omega} \text { for every } i \in S_{k}\right\} \leq\left(2^{1+\delta} e\right)^{-n^{2}},
\end{aligned}
$$

where $k:=n+\lfloor\kappa n / 4\rfloor$.

Proof of Theorem 4.1. Denote by $\mathcal{S}$ the family of all $n+\lfloor\kappa n / 4\rfloor$-element subsets of $S_{m}$ and, for every $S \in \mathcal{S}$, set

$$
\mathcal{A}_{S}=\left\{\omega \in \boldsymbol{\Omega}_{0} \mid \text { there exists } T \in \operatorname{Mix}_{n}(\kappa n, 1)\right. \text { such that }
$$

$$
\left.\left\|T g_{i}\right\|_{B_{\omega}} \leq c^{\prime} \sqrt{n} \text { for every } i \in S\right\} \text {. }
$$

Proposition 4.2 says that $\mathbf{P}\left(\mathcal{A}_{\widetilde{S}}\right) \leq\left(2^{1+\delta} e\right)^{-n^{2}}$. On the other hand, the exchangeability of $g_{i}$ 's implies that $\mathbf{P}\left(\mathcal{A}_{S}\right)$ is the same for all $S \in \mathcal{S}$. Thus

$$
\mathbf{P}\left(\bigcup_{S \in \mathcal{S}} \mathcal{A}_{S}\right) \leq\left(2^{1+\delta} e\right)^{-n^{2}} \operatorname{card} \mathcal{S}<e^{-n^{2}}
$$

Let $\boldsymbol{\Omega}_{1}$ be the set of $\omega$ 's considered in Theorem 4.1. We claim that

$$
\Omega_{0} \backslash \Omega_{1} \subset \bigcup_{S \in \mathcal{S}} \mathcal{A}_{S}
$$

Indeed, let $\omega \in \boldsymbol{\Omega}_{0} \backslash \boldsymbol{\Omega}_{1}$. Then

$$
\operatorname{card}\left\{i \in S_{m} \mid\left\|T g_{i}\right\|_{B_{\omega}}>c^{\prime} \sqrt{n}\right\} \leq\lfloor\delta n / 2\rfloor
$$

for some $T \in \operatorname{Mix}_{n}(\kappa n, 1)$. Since $\kappa \leq \delta$, it follows that $\lfloor(1+\delta) n\rfloor-\lfloor\delta n / 2\rfloor \geq$ $n+\lfloor\kappa n / 4\rfloor$. Hence there exists $\bar{S} \in \mathcal{S}$ such that $\left\|T g_{i}\right\|_{B_{\omega}} \leq c^{\prime}(\kappa, \delta) \sqrt{n}$ for every $i \in \bar{S}$, which means that $\omega \in \mathcal{A}_{\bar{S}}$. Hence, by (4.2), (4.3) and Facts 
3.1-3.3, we get (for $n>1$ )

$$
\mathbf{P}\left(\boldsymbol{\Omega}_{1}\right)=\mathbf{P}\left(\boldsymbol{\Omega}_{0}\right)-\mathbf{P}\left(\boldsymbol{\Omega}_{0} \backslash \boldsymbol{\Omega}_{1}\right) \geq 1-3 e^{-4 n}-e^{-n^{2}}>1-e^{-n} .
$$

5. Proof of Proposition 4.2. In order to make the argument smoother, we shall assume as we may that

$$
(\boldsymbol{\Omega}, \mathbf{P})=\left(\Omega_{1}, \mathbf{P}_{1}\right) \times\left(\Omega_{2}, \mathbf{P}_{2}\right) \times\left(\Omega_{3}, \mathbf{P}_{3}\right)
$$

and we shall write $\omega=\left(\omega_{1}, \omega_{2}, \omega_{3}\right)$. Moreover, we shall assume that $g_{i}(\omega)$ for $i \in S_{n}$ (resp., $i \in \widetilde{S}, i \in S_{m} \backslash S_{k}=S_{m} \backslash S_{n} \backslash \widetilde{S}$ ) depends only on $\omega_{1}$ (resp., $\left.\omega_{2}, \omega_{3}\right)$. Note that, in the notation of Proposition 4.2, $R(\omega)$ depends only on $\omega_{2}$ and so we shall write $R\left(\omega_{2}\right)$ rather than $R(\omega)$ or $R\left(\omega_{1}, \omega_{2}, \omega_{3}\right)$. Similarly, we define

$$
B\left(\omega_{1}, \omega_{3}\right)=\operatorname{symconv}\left\{g_{i}\left(\omega_{0}\right) \mid i \in S_{m} \backslash \widetilde{S}\right\}
$$

for $\omega=\left(\omega_{1}, \omega_{2}, \omega_{3}\right) \in \Omega$. It follows directly from the definitions that for every $\omega=\left(\omega_{1}, \omega_{2}, \omega_{3}\right) \in \boldsymbol{\Omega}$ we have

$$
R\left(\omega_{2}\right) B_{\omega}=R\left(\omega_{2}\right) B\left(\omega_{1}, \omega_{3}\right) .
$$

The proof of Proposition 4.2 is based on two lemmas.

Lemma 5.1. For every $\omega^{\prime}=\left(\omega_{1}^{\prime}, \omega_{2}^{\prime}, \omega_{3}^{\prime}\right) \in \Omega_{0}, T \in \operatorname{Mix}_{n}(\lfloor 3 \kappa n / 4\rfloor, 1)$, and $\alpha>0$ we have

$\mathbf{P}_{2}\left\{\omega_{2} \in \Omega_{2} \mid R\left(\omega_{2}\right) T g_{i}(\widetilde{\omega}) \in \alpha \sqrt{n} R\left(\omega_{2}\right) B_{\widetilde{\omega}}\right.$ for every $\left.i \in \widetilde{S}\right\} \leq(\widetilde{c} \alpha)^{2\lfloor\kappa n / 4\rfloor^{2}}$, where $\widetilde{\omega}=\left(\omega_{1}^{\prime}, \omega_{2}, \omega_{3}^{\prime}\right)$ and $\widetilde{c}=\widetilde{c}(\kappa, \delta)$ depends on $\kappa$ and $\delta$ only.

The technique for the proof of Lemma 5.1 is well known. It suffices to use (5.1) and then, e.g., to follow the line of argument from the proof of Lemma 14 in [MT3]. We leave the details to the reader.

For a fixed $\omega^{\prime}=\left(\omega_{1}^{\prime}, \omega_{2}^{\prime}, \omega_{3}^{\prime}\right) \in \Omega_{0}$ and for every $\alpha>0$ we define

$$
\begin{aligned}
& \widetilde{\mathbf{A}}_{\alpha}=\left\{T \in L\left(\mathbb{R}^{n}\right) \mid T g_{i}\left(\omega^{\prime}\right) \in \alpha B\left(\omega_{1}^{\prime}, \omega_{3}^{\prime}\right) \text { for } i \in S_{n}\right\}, \\
& \mathbf{A}_{\alpha}=\widetilde{\mathbf{A}}_{\alpha} \cap \operatorname{Mix}_{n}(\lfloor 3 \kappa n / 4\rfloor, 1) .
\end{aligned}
$$

In the above notation we have

LEMma 5.2. Given $\omega^{\prime} \in \boldsymbol{\Omega}_{0}$ and $\alpha>0$, the set $\mathbf{A}_{\alpha}$ admits a $1 /(2 C(4))$ net $\mathcal{N}_{\alpha}$ with respect to the operator norm on $L\left(B_{2}^{n}, \alpha B\left(\omega_{1}^{\prime}, \omega_{3}^{\prime}\right)\right)$ with

$$
\operatorname{card} \mathcal{N}_{\alpha} \leq C(\delta)^{n^{2}}
$$

where $C(\delta)>1$ depends on $\delta$ only and $C(4)$ is taken from Fact 3.2.

Proof. Fix $\omega^{\prime} \in \Omega_{0}$ and note that, by Fact 3.2, $\left\|g_{i}\left(\omega^{\prime}\right)\right\|_{2} \leq C(4)$ for $i \in S_{m}$. Hence (cf., e.g., [MT3, Lemma 5])

$$
\operatorname{vol}\left(\alpha B\left(\omega_{1}^{\prime}, \omega_{3}^{\prime}\right)\right) \leq\left(C_{1}(\delta) \alpha / n\right)^{n}
$$

where $C_{1}(\delta)>0$ depends on $\delta$ only. On the other hand, since $n^{-1 / 2} B_{2}^{n} \subset B_{1}^{n}$, we infer that the ellipsoid

$$
\mathcal{E}=\Gamma_{n, m \mid \mathbb{R}^{n}}\left(\alpha n^{-1 / 2} B_{2}^{n}\right)
$$


is contained in $\alpha B\left(\omega_{1}^{\prime}, \omega_{3}^{\prime}\right)$. By Fact 3.1 we have

$$
\operatorname{vol} \mathcal{E} \geq\left(c(\delta) \alpha n^{-1 / 2}\right)^{n} \operatorname{vol} B_{2}^{n} \geq\left(c_{2}(\delta) \alpha / n\right)^{n},
$$

where $c_{2}(\delta)>0$ depends on $\delta$ only. Similarly, by the same fact,

$$
\operatorname{vol} B \geq c(\delta)^{n} \operatorname{vol} B_{1}^{n}
$$

where $B=\operatorname{conv}\left\{ \pm g_{i}\left(\omega^{\prime}\right) \mid i \in S_{n}\right\}$.

Finally, by [MT4, Proposition 5.3] and the estimates above we see that the set $\mathbf{A}_{\alpha}$ of operators admits a $1 /(2 C(4))$-net $\mathcal{N}_{\alpha}$ in the operator norm on $L\left(B_{2}^{n}, \mathcal{E}\right)$ (and therefore in the operator norm on $L\left(B_{2}^{n}, \alpha B\left(\omega_{1}^{\prime}, \omega_{3}^{\prime}\right)\right)$ ) with

$$
\operatorname{card} \mathcal{N}_{\alpha} \leq(2 C C(4))^{n^{2}}\left(\frac{\operatorname{vol}\left(\alpha B\left(\omega_{1}^{\prime}, \omega_{3}^{\prime}\right)\right) \operatorname{vol} B_{1}^{n}}{\operatorname{vol} \mathcal{E} \operatorname{vol} B}\right)^{n} \leq\left(\frac{2 C C(4) C_{1}(\delta)}{c_{2}(\delta) c(\delta)}\right)^{n^{2}}
$$

where $C>1$ is a suitable numerical constant, which completes the proof.

Proof of Proposition 4.2. Fix $\delta>0, \kappa \in(0,1 / 2]$ and $\omega^{\prime}=\left(\omega_{1}^{\prime}, \omega_{2}^{\prime}, \omega_{3}^{\prime}\right)$ $\in \Omega_{0}$. Let $\alpha_{0}>0$ (to be specified later). Again, let $k=n+\lfloor\kappa n / 4\rfloor$ and define

$$
\begin{array}{r}
\mathcal{A}\left(\omega_{1}^{\prime}, \omega_{3}^{\prime}\right):=\left\{\omega_{2} \in \Omega_{2} \mid \text { there exists } T \in \operatorname{Mix}_{n}(\kappa n, 1)\right. \text { such that } \\
\qquad\left(\omega_{2}\right) T g_{i}\left(\omega_{1}^{\prime}, \omega_{2}, \omega_{3}^{\prime}\right) \in \alpha_{0} \sqrt{n} R\left(\omega_{2}\right) B\left(\omega_{1}^{\prime}, \omega_{3}^{\prime}\right) \text { for } i \in S_{k} \\
\text { and } \left.\left(\omega_{1}^{\prime}, \omega_{2}, \omega_{3}^{\prime}\right) \in \Omega_{0}\right\} .
\end{array}
$$

Also, for every $T \in \operatorname{Mix}_{n}(\lfloor 3 \kappa n / 4\rfloor, 1)$ set

$\mathcal{A}\left(\omega_{1}^{\prime}, \omega_{3}^{\prime}, T\right)=\left\{\omega_{2} \in \Omega_{2} \mid R\left(\omega_{2}\right) T g_{i}\left(\omega_{1}^{\prime}, \omega_{2}, \omega_{3}^{\prime}\right) \in 2 R\left(\omega_{2}\right) \alpha_{0} \sqrt{n} B\left(\omega_{1}^{\prime}, \omega_{3}^{\prime}\right)\right.$

for $i \in S_{k}$ and $\left.\left(\omega_{1}^{\prime}, \omega_{2}, \omega_{3}^{\prime}\right) \in \Omega_{0}\right\}$.

Finally, let $\mathcal{N}_{\alpha_{0} \sqrt{n}}$ be the net in $\mathbf{A}_{\alpha_{0} \sqrt{n}}$ from Lemma 5.2.

We claim that

$$
\mathcal{A}\left(\omega_{1}^{\prime}, \omega_{3}^{\prime}\right) \subset \bigcup_{T \in \mathcal{N}_{\alpha_{0} \sqrt{n}}} \mathcal{A}\left(\omega_{1}^{\prime}, \omega_{3}^{\prime}, T\right)
$$

Indeed, pick $\omega_{2} \in \mathcal{A}\left(\omega_{1}^{\prime}, \omega_{3}^{\prime}\right)$ and $T \in \operatorname{Mix}_{n}(\kappa n, 1)$ such that

$$
R\left(\omega_{2}\right) T g_{i}\left(\omega_{1}^{\prime}, \omega_{2}, \omega_{3}^{\prime}\right) \in R\left(\omega_{2}\right) \alpha_{0} \sqrt{n} B\left(\omega_{1}^{\prime}, \omega_{3}^{\prime}\right)
$$

for $i \in S_{k}=S_{n} \cup \widetilde{S}$. Note that, by a standard "lifting" argument, the condition (5.3) yields the existence of $T_{1} \in L\left(\mathbb{R}^{n}\right)$ satisfying

$$
T_{1} g_{i}\left(\omega_{1}^{\prime}, \omega_{2}, \omega_{3}^{\prime}\right) \in \alpha_{0} \sqrt{n} B\left(\omega_{1}^{\prime}, \omega_{3}^{\prime}\right) \quad \text { for every } i \in S_{n}
$$

and

$$
R\left(\omega_{2}\right) T_{1}=R\left(\omega_{2}\right) T
$$

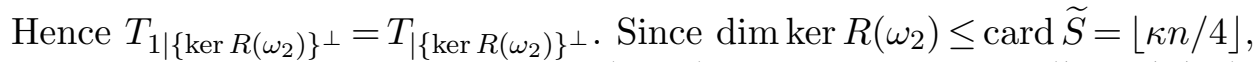
it is easy to verify that $T \in \operatorname{Mix}_{n}(\kappa n, 1)$ implies $T_{1} \in \operatorname{Mix}_{n}(\lfloor 3 \kappa n / 4\rfloor, 1)$. Thus $T_{1} \in \mathbf{A}_{\alpha_{0} \sqrt{n}}$. Pick $T_{0} \in \mathcal{N}_{\alpha_{0} \sqrt{n}}$ with

$$
\left\|\left(T_{1}-T_{0}\right): B_{2}^{n} \rightarrow \alpha_{0} \sqrt{n} B\left(\omega_{1}^{\prime}, \omega_{3}^{\prime}\right)\right\| \leq 1 /(2 C(4))
$$


and an arbitrary $i \in \widetilde{S}$. Since $\left(\omega_{1}^{\prime}, \omega_{2}, \omega_{3}^{\prime}\right) \in \boldsymbol{\Omega}_{0}$, it follows that $\left\|g_{i}\left(\omega_{1}^{\prime}, \omega_{2}, \omega_{3}^{\prime}\right)\right\|_{2}$ $\leq C(4)$ (Fact 3.2). Thus by (5.3)-(5.6) we get

$$
\begin{aligned}
R\left(\omega_{2}\right) T_{0} g_{i}\left(\omega_{1}^{\prime}, \omega_{2}, \omega_{3}^{\prime}\right) & \\
& =R\left(\omega_{2}\right) T_{1} g_{i}\left(\omega_{1}^{\prime}, \omega_{2}, \omega_{3}^{\prime}\right)+R\left(\omega_{2}\right)\left(T_{0}-T_{1}\right) g_{i}\left(\omega_{1}^{\prime}, \omega_{2}, \omega_{3}^{\prime}\right) \\
& \in R\left(\omega_{2}\right) T_{1} g_{i}\left(\omega_{1}^{\prime}, \omega_{2}, \omega_{3}^{\prime}\right)+\left(\alpha_{0} \sqrt{n} / 2\right) R\left(\omega_{2}\right) B\left(\omega_{1}^{\prime}, \omega_{3}^{\prime}\right) \\
& =R\left(\omega_{2}\right) T g_{i}\left(\omega_{1}^{\prime}, \omega_{2}, \omega_{3}^{\prime}\right)+\left(\alpha_{0} \sqrt{n} / 2\right) R\left(\omega_{2}\right) B\left(\omega_{1}^{\prime}, \omega_{3}^{\prime}\right) \\
& \subset 2 \alpha_{0} \sqrt{n} R\left(\omega_{2}\right) B\left(\omega_{1}^{\prime}, \omega_{3}^{\prime}\right) .
\end{aligned}
$$

Thus

$$
R\left(\omega_{2}\right) T_{0} g_{i}\left(\omega_{1}^{\prime}, \omega_{2}, \omega_{3}^{\prime}\right) \subset 2 \alpha_{0} \sqrt{n} R\left(\omega_{2}\right) B\left(\omega_{1}^{\prime}, \omega_{3}^{\prime}\right)
$$

for every $i \in \widetilde{S}$. Recall that $g_{i}\left(\omega_{1}, \omega_{2}, \omega_{3}\right)$ for every $i \in S_{n}$ depends on $\omega_{1} \in \Omega_{1}$ only. Therefore, $g_{i}\left(\omega_{1}^{\prime}, \omega_{2}^{\prime}, \omega_{3}^{\prime}\right)=g_{i}\left(\omega_{1}^{\prime}, \omega_{2}, \omega_{3}^{\prime}\right)$ for every $i \in S_{n}$ and every $\omega_{2} \in \Omega_{2}$. Since $T_{0} \in \mathcal{N}_{\alpha_{0} \sqrt{n}} \subset \mathbf{A}_{\alpha_{0} \sqrt{n}}$, we infer that (5.7) holds for every $i \in S_{n}$ as well. Thus $\omega_{2} \in \mathcal{A}\left(\omega_{1}^{\prime}, \omega_{3}^{\prime}, T_{0}\right)$, which concludes the proof of $(5.2)$.

Returning to the proof of Proposition 4.2 note that for each $\omega_{0} \in \boldsymbol{\Omega}_{0}$, Lemmas 5.1, 5.2 and (5.2) yield

$$
\begin{aligned}
\mathbf{P}_{2}\left(\mathcal{A}\left(\omega_{1}^{\prime}, \omega_{3}^{\prime}\right)\right) & \leq \sum_{T \in \mathcal{N}_{\alpha_{0} \sqrt{n}}} \mathbf{P}_{2}\left(\mathcal{A}\left(\omega_{1}^{\prime}, \omega_{3}^{\prime}, T\right)\right) \\
& \leq C(\delta)^{n^{2}}\left(2 \widetilde{c}(\kappa, \delta) \alpha_{0}\right)^{2\lfloor\kappa n / 4\rfloor^{2}} .
\end{aligned}
$$

By choosing $\alpha_{0}=\alpha_{0}(\kappa, \delta)$ sufficiently small we may make the right hand side less than $\left(2^{1+\delta} e\right)^{-n^{2}}$. In view of (5.1), the proof is completed by setting $c^{\prime}(\kappa, \delta)=\alpha_{0}(\kappa, \delta)$ and integrating (5.8) with respect to $\omega_{1}^{\prime}$ and $\omega_{3}^{\prime}$.

\section{References}

[B] K. Ball, Normed spaces with a weak-Gordon-Lewis property, in: Functional Analysis (Austin, TX, 1987/1989), Lecture Notes in Math. 1470, Springer, Berlin, 1991, 36-47.

[BKPS] P. Billard, S. Kwapień, A. Pełczyński and C. Samuel, Biorthogonal systems of random unconditional convergence in Banach spaces, in: Texas Functional Analysis Seminar 1985-1986 (Austin, TX, 1985-1986), Longhorn Notes, Univ. Texas, Austin, 1986, 13-35.

[Bo] J. Bourgain, On finite-dimensional homogeneous Banach spaces, in: GAFA Israel Seminar 1986-87, Lecture Notes in Math. 1317, Springer, 1988, 232-239.

[DS] K. R. Davidson and S. J. Szarek, Local operator theory, random matrices and Banach spaces, in: Handbook on the Geometry of Banach Spaces, Vol. 1, W. B. Johnson and J. Lindenstrauss (eds.), Elsevier, 2001, 317-366.

[FKP] T. Figiel, S. Kwapień and A. Pełczyński, Sharp estimates for the constants of local unconditional structure of Minkowski spaces, Bull. Acad. Polon. Sci. 25 (1977), 1221-1226. 
[G] E. D. Gluskin, The diameter of Minkowski compactum is roughly equal to $n$, Funct. Anal. Appl. 15 (1981), no. 1, 57-58 (English transl.).

[K] B. S. Kashin, The widths of certain finite-dimensional sets and classes of smooth functions, Izv. Akad. Nauk SSSR Ser. Mat. 41 (1977), 334-351 (in Russian).

[M1] P. Mankiewicz, Finite-dimensional Banach spaces with symmetry constant of order $\sqrt{n}$, Studia Math. 79 (1984), 193-200.

[M2] - Compact groups of operators on proportional quotients of $l_{1}^{n}$, Israel J. Math. 109 (1999), 75-91.

[MT1] P. Mankiewicz and N. Tomczak-Jaegermann, A solution of the finite-dimensional homogeneous Banach space problem, Israel J. Math. 75 (1991), 129-159.

[MT2] - - - Schauder bases in subspaces of quotients of $l_{2}(X)$, Amer. J. Math. 116 (1994), 1341-1363.

[MT3] -, -, Quotients of finite-dimensional Banach spaces; random phenomena, in: Handbook on the Geometry of Banach Spaces, Vol. 2, W. B. Johnson and J. Lindenstrauss (eds.), Elsevier, to appear.

[MT4] - - - Geometry of families of random projections of symmetric convex bodies, Geom. Funct. Anal. 11 (2001), 1282-1326.

[Mi1] V. D. Milman, A new proof of the theorem of A. Dvoretzky on sections of convex bodies, Funct. Anal. Appl. 5 (1971), no. 4, 28-37 (English transl.).

[Mi2] - Randomness and pattern in convex geometric analysis, in: Proc. Internat. Congress Math., Vol. II (Berlin, 1998), Doc. Math. 1998, Extra Vol. II, 665-677.

[MiS] V. D. Milman and G. Schechtman, Global versus local asymptotic theories of finite-dimensional normed spaces, Duke Math. J. 90 (1997), 73-93.

[S1] S. J. Szarek, On Kashin's almost Euclidean orthogonal decomposition of $\ell_{1}^{n}$, Bull. Acad. Polon. Sci. 26 (1978), 691-694.

[S2] - The finite dimensional basis problem with an appendix on nets of Grassmann manifolds, Acta Math. 151 (1983), 153-179.

[S3] - On the existence and uniqueness of complex structure and spaces with "few" operators, Trans. Amer. Math. Soc. 293 (1986), 339-353.

[S4] - Spaces with large distance to $\ell_{\infty}^{n}$ and random matrices, Amer. J. Math. 112 (1990), 899-942.

[S5] - Condition numbers of random matrices, J. Complexity 7 (1991), 131-149.

[T] N. Tomczak-Jaegermann, Banach-Mazur Distances and Finite-Dimensional Operator Ideals, Pitman Monogr. Surveys Pure Appl. Math. 38, Longman, Harlow, 1989.

Institute of Mathematics

Polish Academy of Sciences

Śniadeckich 8

00-950 Warszawa, Poland

E-mail: piotr@impan.gov.pl
Équipe d'Analyse Fonctionnelle Université Paris VI, B.C. 186 4, Place Jussieu 75252 Paris, France E-mail: szarek@ccr.jussieu.fr

Department of Mathematics Case Western Reserve University Cleveland, OH 44106-7058, U.S.A.

Received October 29, 2001 